\title{
Clinical effects of miR-101 on prognosis of hepatocellular carcinoma and carcinogenic mechanism of anti-miR-101
}

\author{
XUECHENG LV ${ }^{1}$, JINGHUA $\mathrm{LI}^{2}$ and BINGNAN YANG ${ }^{3}$ \\ ${ }^{1}$ Department of Pharmacy, The First People's Hospital of Shangqiu, Shangqiu, Henan 476100; \\ Departments of ${ }^{2}$ Cardiovascular Surgery and ${ }^{3}$ Neurosurgery, Henan Provincial People's Hospital, \\ Zhengzhou, Henan 450000, P.R. China
}

Received March 3, 2016; Accepted July 11, 2016

DOI: $10.3892 /$ or.2016.4980

\begin{abstract}
The aim of this study was to verify whether anti-miR-101 participates in the treatment of hepatocellular carcinoma (HCC) as a small-molecule antitumor agent, and to explore the effect on phosphatase and tensin homolog deleted on chromosome 10 (PTEN). Patients who received consecutive hepatectomies were followed-up, and miR-101 expressions in their tumor and paracancerous tissues were detected. Correlation between miR-101 expression and clinical pathological factors and prognosis was studied. High-throughput sequencing was used to detect the genetic and microRNA (miRNA) levels of tumor tissues. Expression of anti-miR-101 in different HCC cell lines was determined, and those of desired genes and proteins were detected by qRT-PCR and western blotting to obtain the target gene. miR-101 was significantly upregulated in HCC patients compared with that in paracancerous tissues. High miR-101 expression, vascular invasion, tumor size $\geq 7 \mathrm{~cm}$ and late pathological stage were the risk factors of recurrence-free survival rate. High miR-101 expression was the independent prognostic factor of total and recurrence-free survival rates. CXCL12, IL6R, FOXO3 and PTEN were screened as desired genes, and only PTEN was expressed significantly differently in three cell lines. miR-101 could bind 3'-UTR of WT-PTEN with reduced fluorescent intensity, suggesting that PTEN was the target gene. SMMC7721, HepG2 and Huh7 were eligible cell lines for miR-101 studies. miR-101 was an applicable molecular marker of HCC. Anti-miR-101 regulated the transcription of PTEN and may promote cell proliferation, differentiation and apoptosis by regulating downstream genes with PTEN. The regulatory effects of anti-miR-101 on PTEN provide valuable evidence for finding novel miRNA drugs.
\end{abstract}

Correspondence to: Dr Bingnan Yang, Department of Neurosurgery, Henan Provincial People's Hospital, Zhengzhou, Henan 450000, P.R. China

E-mail: yangbndn@126.com

Key words: microRNA-101, phosphatase and tensin homolog deleted on chromosome 10, hepatocellular carcinoma, oncogene

\section{Introduction}

Hepatocellular carcinoma (HCC) is one of the fatal cancers threatening people worldwide, with over half of them being Chinese. Late diagnosis of HCC leads to poor prognosis by restricting the selection of treatment protocols. Therefore, finding tumor markers for early diagnosis is crucial $(1,2)$. Although AFP is an important marker of detecting and monitoring $\mathrm{HCC}$, its individual detection in small $\mathrm{HCC}$ patients has a $40 \%$ chance of indicating false negative. Thus, it is imperative to find other markers that are more sensitive and earlier detectable to clarify the development mechanism of HCC and to improve therapeutic effects and prognosis (3).

MicroRNA (miRNA), as a non-protein-coding RNA family that can negatively regulate gene expression, can repress protein translation or induce RNA degradation by interacting with mRNA. miRNAs also function as effective small-molecule drugs. Mature miRNA, which is a single-strand RNA molecule length of about $19025 \mathrm{nt}$, can inhibit the translation of target gene and participate in cell differentiation, proliferation and apoptosis by binding 3'-UTR of mRNA through complete or incomplete base pairing (4). The miRNA-101 expression profiles of tumor cells and paracancerous tissues differ significantly, thus being able to distinguish HCC (5), breast carcinoma $(6,7)$, lung carcinoma (8), pancreatic carcinoma (9) and leukemia (10) from adjoining normal tissues.

We have previously found that miR-101 was evidently highly expressed in HCC patients, which is associated with the unique regulatory effects of miRNA on phosphatase and tensin homolog deleted on chromosome 10 (PTEN) reported by Karreth et al $(11,12)$. Although anti-miR-101 and PTEN have previously been correlated, in vitro carcinogenesis experiment has seldom been combined with clinical study results. Thereby motivated, we explored the influence of miR-101 on the onset of HCC as an oncogene and its direct effect on PTEN, aiming to verify the results of Karreth et al, miRNA and gene expression profiles in tumor and paracancerous tissues were detected. The correlation between differentiated anti-miR-101 expressions and malignant behavior of HCC, prognosis, as well as total and recurrence-free survival rates were summarized. The target gene corresponding to miR-101 was determined to preliminarily postulate the role of miR-101 in the onset and development of HCC. The results indicate anti-miR-101 is a 
feasible marker for the diagnosis and prognosis evaluation of HCC.

\section{Materials and methods}

Compliance with ethical requirements. Written consent was received from all the patients. All information and sample collection as well as experimental protocols accorded with the 'Ethics Committee Standard Operating Procedures (SOP)', and all the experiments have been approved by the Ethics Committee of our hospital.

General information and tissue samples. Seventy-eight HCC patients who received consecutive hepatectomies in our hospital from January 2009 to June 2015 were selected. Inclusion criteria: i) Isolated or multiple tumors (no more than 3); ii) without portal vein, hepatic vein or postcava tumor thrombi; iii) without extrahepatic metastasis; iv) Child-Pugh scores ranging between 5 and 7; and v) first visit patients who had not received any transcatheter arterial chemoembolization, local treatment or radiotherapy $(13,14)$. Tumor and the corresponding paracancerous tissues were subjected to RNA later treatment after resection, archived and stored in tissue bank, and stored at $-80^{\circ} \mathrm{C}$. No tumor tissues succumbed to necrosis, and normal hepatic tissues located $3 \mathrm{~cm}$ from the edge of tumor tissues were used as paracancerous tissues. Tissues of each case were sampled in triplicate to prevent potential contamination. Clinical data were collected from the medical records, and no patients had received chemotherapy prior to the surgeries. Written consent was received from all the patients. All of them were diagnosed as HCC, as confirmed by H\&E staining.

The patients aged 48-82 years (median, 53 years) included 63 males $(80.8 \%)$ and 15 females $(19.2 \%)$. There were 61 serum HBsAg-positive cases (78.2\%), and serum AFP levels were 0.78-115600 $\mu \mathrm{g} / \mathrm{l}$ (median, $230 \mathrm{pg} / \mathrm{l})$. Fifty-eight cases were complicated with cirrhosis $(74.4 \%)$, and the others were not (25.6\%). Maximum tumors were sized 1.4-15.4 cm (median, $7.3 \mathrm{~cm}$ ). Tumor differentiation degrees were determined according to Edmondson-Steiner grading standard (40 cases of grade I and II, 38 cases of grade III and IV) (15). The patients were classified into 37 cases of stage I, 24 cases of stage II and 17 cases of stage III according to the TNM staging standard stipulated by UICC and AJCC (16). They were last followed-up on 30th June, 2015.

Cell lines. Human hepatocarcinoma cell lines HEPG2, HEP3B, Sk-Hepl, Huh7, SMMC-7721, PLC, MHCC-97H and MHCC-97L were purchased from Zhongyuan Union Stem Cell Bioengineering Co. (Zhejiang, China). Immortalized hepatocytes L-02 and human kidney epithelial cells 293T were provided by the Molecular Biotechnology Center of our hospital.

Main reagents and solutions. miRNA expression vector anti-miR-101 (inhibitor), pre-miR-101 (mimics) and control miR-NC were purchased from Ambion (Carlsbad, CA, USA). Wild-type pcDNA 3.1-PTEN-WT and mutant pcDNA 3.1-PTEN-MU plasmids were purchased from GeneCopoeia, Inc. (Rockville, MD, USA). Luciferase reporter assay vector pGL3M, pcDNA3.1 plasmid, EcoRI and $X b a I$ were obtained from Promega Corp. (Madison, WI, USA). Lipofectamine ${ }^{\mathrm{TM}} 2000$ liposome, E. coli strain DH5a and quantitative PCR kit Platinum ${ }^{\circledR}$ SYBR $^{\circledR}$ Green qPCR SuperMix-UDG reagent were purchased from Invitrogen (Carlsbad, CA, USA). Dual-luciferase reporter assay kit Dual-Light $^{\circledR}$ system (P/N T1003) was obtained from Applied Biosystems Life Technologies (Foster City, CA, USA). B-type ultrapure plasmid extraction kit was bought from BioDev-Tech Co. (Beijing, China). Restriction endonuclease HindIII and SpeI, as well as DNA T4 ligase were purchased from New England BioLabs, Inc. (Ipswich, MA, USA). The First Strand cDNA Synthesis kit was purchased from Fermentas (Waltham, MA, USA). Western blotting-related polyclonal antibodies were obtained from Cell Signaling Technology, Inc. (Danvers, MA, USA). Other reagents were purchased from SigmaAldrich (St. Louis, MO, USA) or Sinopharm Chemical Reagent Co., Ltd. (Shanghai, China) (analytically pure). Primers were designed and synthesized by Shanghai Sangon Biological Engineering Technology and Services Co., Ltd. (Shanghai, China).

Cell culture. All cell lines were cultured in high-glucose Dulbecco's modified Eagle's medium (DMEM) containing $10 \%$ fetal bovine serum, and were incubated in a $5 \% \mathrm{CO}_{2}$ incubator at $37^{\circ} \mathrm{C}$ with saturated humidity. After being digested with $0.25 \%$ trypsin, the cells growing to $80-90 \%$ confluence were then passaged. The cells growing logarithmically were finally used (17).

Total RNA extraction. Total RNA was extracted by TRIzol reagent according to the manufacturer's instructions. The tumor and paracancerous tissues of 9 cases were compared by digital miRNA expression profiling. They were then classified into stage I (4 cases), stage II (3 cases) and stage III ( 2 cases) (16), including 7 males and 2 females. Culture medium was discarded after cells fully adhered to the bottom, to which $1 \mathrm{ml}$ of TRIzol reagent was added per $0.5-1 \times 10^{7}$ cells. The following procedure was the same as that of tissue RNA extraction (18).

Gene and digital miRNA expression profiling. Illumina HiSeq $^{\mathrm{TM}} 2000$ high-throughput small-RNA sequencing and synthesis were performed simultaneously, aiming to minimize the secondary structure-induced local missing. The screened high-quality sequences were classified and annotated to obtain information on components and expression levels. All miRNA segments were annotated, and target gene prediction was conducted for new miRNA $(19,20)$.

Detection of hsa-miR-101 expression levels before and after transfecting miRNA precursor by $q R T-P C R$. miRNA precursor was transfected by the RNA transient transfection method according to the instruction of Lipofectamine ${ }^{\mathrm{TM}} 2000$ (Invitrogen). Diluted anti-miR-101, pre-miR-101 and miR-NC were mixed with liposomes in culture medium. Total RNA was extracted by mirVana ${ }^{\mathrm{TM}}$ miRNA isolation kit 24 and $48 \mathrm{~h}$ later to measure miR-101 expression levels. After RNA purification, cDNA was subjected to reverse transcription with the reaction systems and primer sequences reported 
Table I. Digital miRNA expression profiling results.

\begin{tabular}{|c|c|c|c|c|c|}
\hline \multicolumn{3}{|c|}{ Upregulated miRNAs } & \multicolumn{3}{|c|}{ Downregulated miRNAs } \\
\hline miRNA & Difference fold & q-value (\%) & miRNA & Difference fold & q-value $(\%)$ \\
\hline hsa-miR-101 & 4.733 & 0 & hsa-miR-486 & 0.269 & 0 \\
\hline hsa-miR-30e & 4.265 & 0 & hsa-miRPlus-E1012 & 0.304 & 0 \\
\hline hsa-miR-221 & 4.038 & 0 & hsa-miR-223 & 0.366 & 0 \\
\hline hsa-miR-23a & 3.726 & 0 & hsa-miR-766 & 0.396 & 0 \\
\hline hsa-miR-16 & 3.714 & 0 & hsa-miR-608 & 0.405 & 0 \\
\hline hsa-miR-30a & 3.284 & 0 & hsa-miRPIUS-El120 & 0.417 & 0 \\
\hline hsa-miR-21 & 3.197 & 0 & hsa-miR-129 & 0.433 & 0 \\
\hline hsa-miR-15a & 3.006 & 0 & hsa-miRPlus-A1027 & 0.451 & 0 \\
\hline hsa-miR-24 & 2.994 & 0 & hsa-miR-625 & 0.470 & 0 \\
\hline hsa-miR-195 & 2.754 & 0 & hsa-miRPlus-F1205 & 0.476 & 0 \\
\hline hsa-miR-200a & 2.732 & 0 & hsa-miR-665 & 0.514 & 0 \\
\hline hsa-miR-125a & 2.525 & 0 & hsa-miR-767-3p & 0.548 & 0 \\
\hline hsa-miR-222 & 2.471 & 0 & hsa-miRPlus-F1208 & 0.557 & 0 \\
\hline hsa-miR-205 & 2.378 & 0 & hsa-miR-634 & 0.565 & 0 \\
\hline hsa-miR-30b & 2.236 & 0 & hsa-miRPlus-E124 & 0.594 & 0 \\
\hline hsa-miR-378 & 1.941 & 0 & hsa-miRPlus-18b & 0.608 & 0 \\
\hline hsa-miR-27a & 1.879 & 0 & & & \\
\hline hsa-miR-142-3p & 1.677 & 0 & & & \\
\hline hsa-miR-497 & 1.524 & 0 & & & \\
\hline
\end{tabular}

Tumor and paracancerous tissues of 9 cases were compared.

previously (21-25). Primer sequences: miR-101 upstream, 5'-TCAC ACTATATCACATTGCCAGG-3' and downstream, 5'-TATGG TTGTTCTGCTCTCTGTCTC-3'; CXCL12 upstream, 5'-CAG TCAACCTGGGCAAAGCC-3' and downstream, 5'-CCTGAG AGTCCTTTTGCGGG-3'; IL6R upstream, 5'-GGCAACCGA GCAAGACTCTC-3' and downstream, 5'-GCGAGGACAGAA GATTTG-3'; FOXO3 upstream, 5'-CTTAAGGATAAGGGC GACA-3' and downstream, 5'-CGACTATGCAGTGACAGG TTG-3'; PTEN upstream, 5' - AG ACAGATC T GT GG GG T GC G GGGTAGGAGT-3' and downstream, 5'-AGACAAGCTTGAC GAAGAGGAGGCGAGA-3'; internal reference U6 upstream, 5'-ATTGGAACGATACAGAGAAGATT-3' and downstream, 5'-GGAACGCTTCACGAATTTG-3'; internal reference GAPDH upstream, 5'-GTCAGTGGTGGACCTGACCT-3' and downstream, 5'-TGAGGAGGGGAGATFCAGTG-3'.

Western blotting. Cells collected at different time intervals were lysed, from which cytoplasm and nucleus protein supernatants were extracted for SDS-PAGE at $120 \mathrm{~V}$ for $2 \mathrm{~h}$. Then the products were electrotransferred onto PVDF membrane and put in TBST blocking buffer containing 5\% defatted milk at room temperature for $1 \mathrm{~h}$. After TBST washing, primary antibody was added, and then the membrane was shaken at $4^{\circ} \mathrm{C}$ overnight. After several times of TBST washing, the membrane was shaken at room temperature for $1 \mathrm{~h}$ after adding rabbit anti-human polyclonal antibody, washed three times and colored by ECL (26).
Plasmid transfection and luciferase reporter assay. Empty vector pcDNA3.1 and luciferase reporter vector pGL3M, as well as recombinant reporter vector pcDNA 3.1-PTEN-WT (or MUT/NC) were transfected with competent host bacteria DH5a. Plasmids were extracted according to the manufacturer's instructions, identified by $\mathrm{Xba \textrm {I }}$ and $E c o \mathrm{RI}$ double digestion, and sequenced by Hohhot Mole Chemical Reagent Co., Ltd. (Hohhot, China) (27).

With the transfection method mediated by the Lipofectamine 2000 liposome, 293T cells were transfected with pGL3M-PTEN-3'UTR (50 ng) and control plasmid pcDNA3.1 (10 ng), and referred to as experimental group pGL3M-WT-PTEN-3'UTR, positive control group pGL3M-MUT-PTEN-3'UTR and negative control group pGL3M. Dual-luciferase reporter assay results were expressed as the ratio of firefly luciferase activity to Renilla luciferase activity.

Statistical analysis. Digital gene and miRNA expression profiles were analyzed by non-monitoring clustering with SAM and TIGR Multiple Array Viewer software package (TMeV version 4.0). Real-time fluorescent quantitative PCR was performed by sequence detection system (SDS) 2.3, and miRNA expression levels were expressed as $\Delta \mathrm{Ct}$ values (Ct-miRNA-Ct U6). Biological data were expressed as mean $\pm \mathrm{SD}$, and the differences between two groups were compared by Student's t-test. Numeration data were compared by Chi-square test or Fisher's exact test. Survival time was 

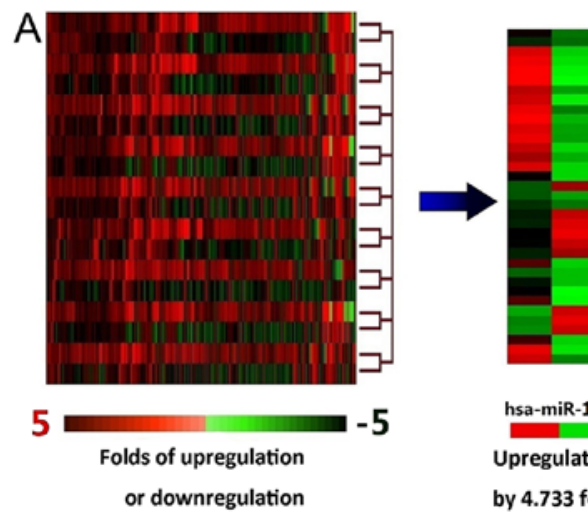

hsa-miR-101
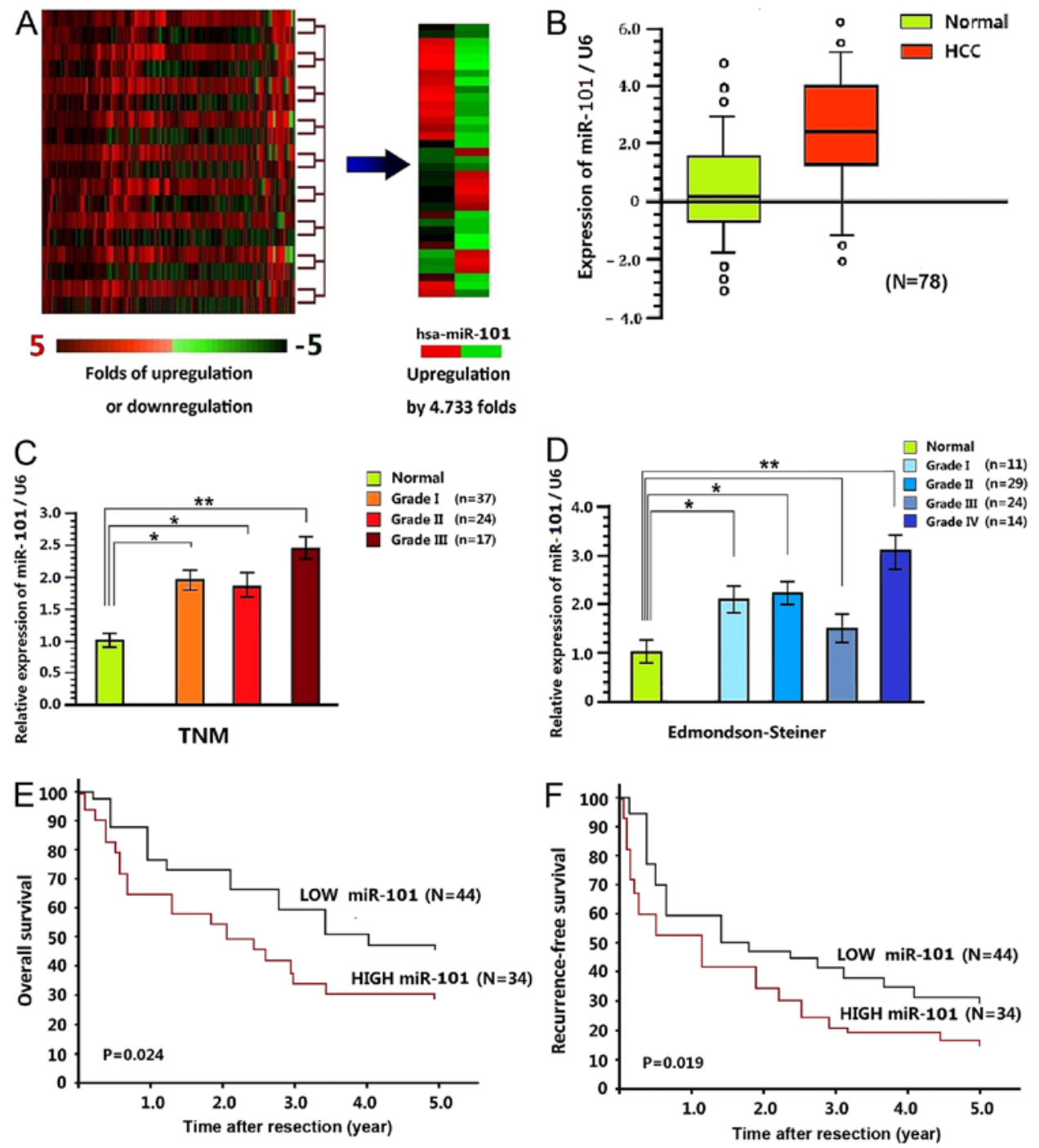

Figure 1. Correlation between miR-101 expression in hepatocellular carcinoma (HCC) tissues, clinical pathological factors and prognosis. (A) miR-101 expression was significantly upregulated in tumor tissues (red, upregulation; green, downregulation); (B) medians of paracancerous and tumor tissues are 0.18 and 2.47, respectively $(\mathrm{P}<0.001)$; $(\mathrm{C})$ miR-101 expressions in tumor tissues in three TNM stages are all higher than those in the corresponding paracancerous tissues; (D) miR-101 expression in tumor tissues in four Edmondson-Steiner stages are all higher than those in paracancerous tissues; (E) 5-year overall survival rate of low miR-101 was decreased from 48.2 to $7.2 \%$, and that of high miR-101 expression group was reduced from 64.7 to $30.6 \%$ ( $\mathrm{P}=0.024$ ); (F) recurrence-free survival rate of low miR-221 expression group was decreased from 59.7 to $32.5 \%$, and that of high expression group was reduced from 52.3 to $17.7 \%(\mathrm{P}=0.019) .{ }^{* *} \mathrm{P}<0.01,{ }^{*} \mathrm{P}<0.05$.

calculated by month, and overall survival time was calculated by that from surgery to death or last follow-up. Survival rate was calculated by Kaplan-Meier method. Single variables were subjected to log-rank test, and significant variables were analyzed by introducing the Cox's model. $\mathrm{P}<0.05$ was considered statistically significant. Data were analyzed by SPSS 15.0 .

\section{Results}

Correlation between miR-101 expression in HCC tissues, clinical pathological factors and prognosis. The miRNAs with significantly changed expressions were screened by digital miRNA expression profiling (Table I). Obviously, the miR-101 expression was significantly upregulated in tumor tissues (Fig. 1A), with the medians of paracancerous and tumor tissues as 0.18 and 2.47 , respectively $(\mathrm{P}<0.001)$ (Fig. 1B). Of the 78 tumor tissues, there were 37 cases of stage I, 24 cases of stage II and 17 cases of stage III based on the TNM grading standard, with their miR-101 expression all higher than those in the corresponding paracancerous tissues (miR-101 expressions were upregulated by 1.96-fold, 1.88-fold and 2.47-fold in the three stages, respectively). Particularly, the expression levels in stage II and III differed significantly between the two groups. According to the Edmondson-Steiner grading standard, there were 11 cases of stage I, 29 cases of stage II, 24 cases of stage II and 14 cases of stage IV, of which miR-101 expression was upregulated by 2.15 -fold, 2.28 -fold, 1.53 -fold and 3.14-fold (Fig. 1D).

The correlations between miR-101 expression and clinical pathological factors, including gender, age, cirrhosis history, HBsAg state, serum AFP level, Child-Pugh grade, vascular invasion status, tumor size, tumor number, tumor capsule, tumor differentiation degree (Edmondson-Steiner grade) and TNM stage, were analyzed. The 78 patients were divided into a low miR-101 expression group and a high miR-101 expression group based on the average upregulation fold (2.47). First, 


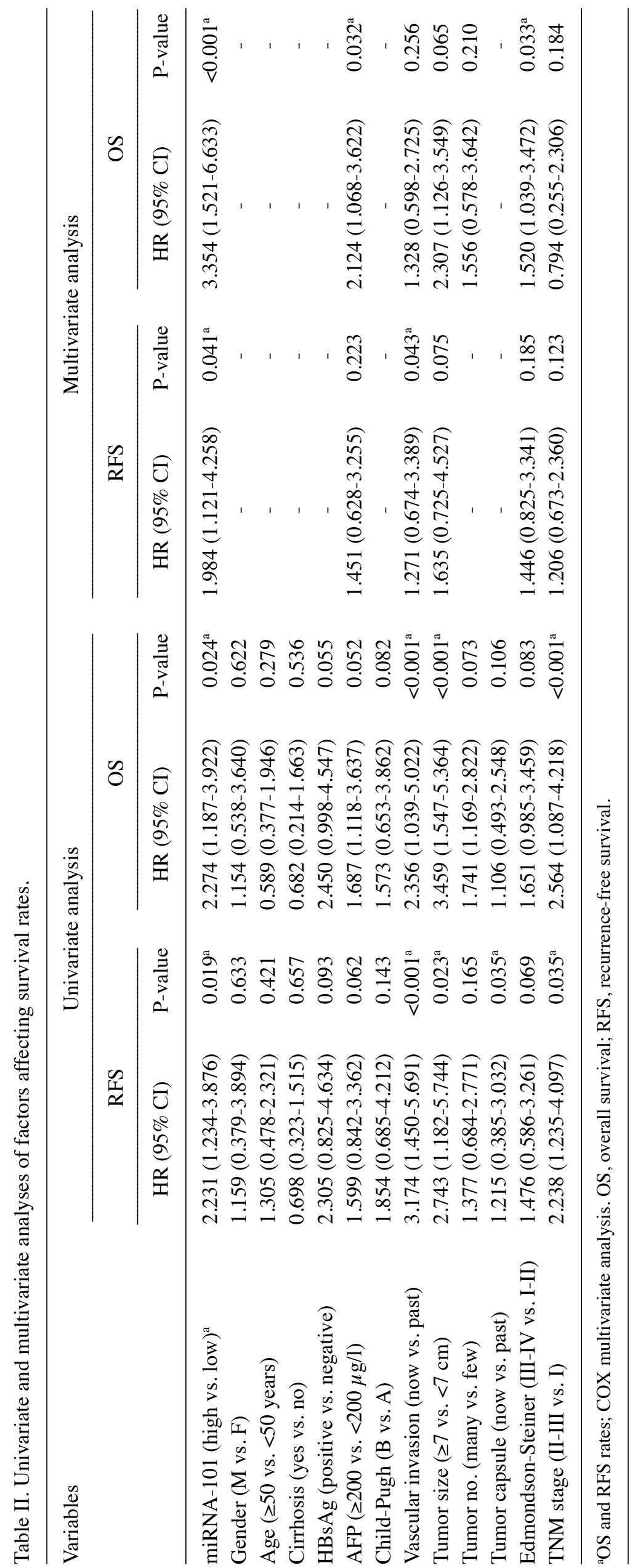



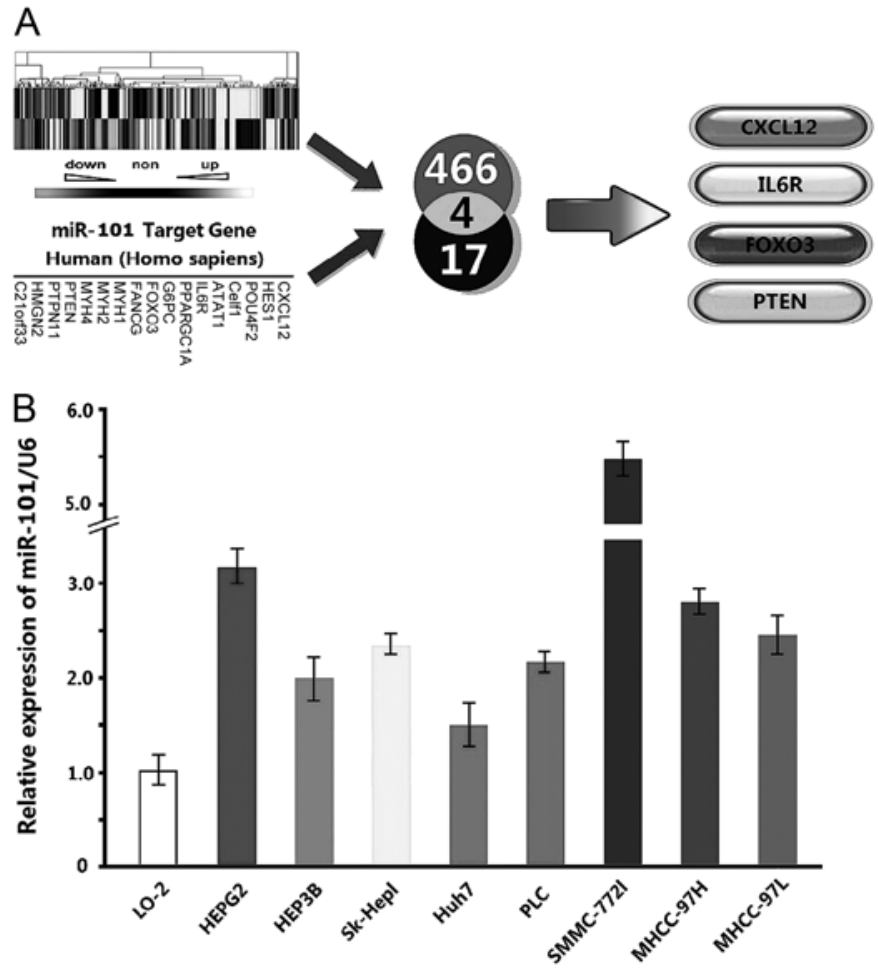

univariate analysis and log-rank test were performed. High miR-101 expression $(\mathrm{P}=0.019)$, vascular invasion $(\mathrm{P}<0.001)$, tumor size $\geq 7 \mathrm{~cm}(\mathrm{P}=0.023)$, tumor capsule $(\mathrm{P}=0.035)$ and late stage (TNM II-III) $(\mathrm{P}=0.035)$ were the risk factors of overall survival rate. Besides, high miR-101 expression $(\mathrm{P}=0.024)$, vascular invasion $(\mathrm{P}<0.001)$, tumor size $\geq 7 \mathrm{~cm} \quad(\mathrm{P}<0.001)$ and late stage (TNM II-III) $(\mathrm{P}=0.001)$ were the risk factors of recurrence-free survival rate (Table II). Multivariate analysis showed that high miR-101 level $(\mathrm{P}=0.041)$ and vascular invasion $(\mathrm{P}=0.043)$ were the risk factors of overall survival rate. High miR-101 expression level $(\mathrm{P}<0.001)$, AFP $\geq 200 \mu \mathrm{g} / \mathrm{l}(\mathrm{P}=0.032)$ and Edmondson-Steiner III-IV stages $(\mathrm{P}=0.033)$ were the risk factors of recurrence-free survival rate (Table II).

Cox multiple regression analysis showed that high miR-101 expression level was the independent prognostic factor of overall and recurrence-free survival rates of HCC patients [OS, (HR, 2.274; 95\% CI, 1.187-3.922); RFS, (HR, 2.231; 95\% CI, 1.234-3.876)]. The 1-, 2-, 3-, 4- and 5-year overall survival rates of low miR-101 expression group were 77.2, 74.3, 60.2\%, 51.9 and $48.2 \%$, respectively, and those of high miR-101 expression group were 64.7, 55.0, 34.1, 30.6 and 30.6\%, respectively $(\mathrm{P}=0.024)$ (Fig. 1E). The 1-, 2-, 3-, 4- and 5-year recurrence-free survival rates of low miR-221 expression group were 59.7, 47.2, 41.0, 34.9 and $32.5 \%$, and those of the high expression group were 52.3, 34.4, 20.4, 19.6 and $17.7 \%$ $(\mathrm{P}=0.019)$ (Fig. 1F).

Screening of miR-101 target gene and expression in different $H C C$ cell lines. A total of 466 genes underwent significantly different changes according to the results of digital gene expression profiling, and CXCL12, HES1, POU4F2, Celf1, ATAT1, IL6R, PPARGC1A, G6PC, FOXO3, FANCG, MYH1, MYH2, MYH4, PTEN, PTPN11, HMGN2 and C21orf33 were

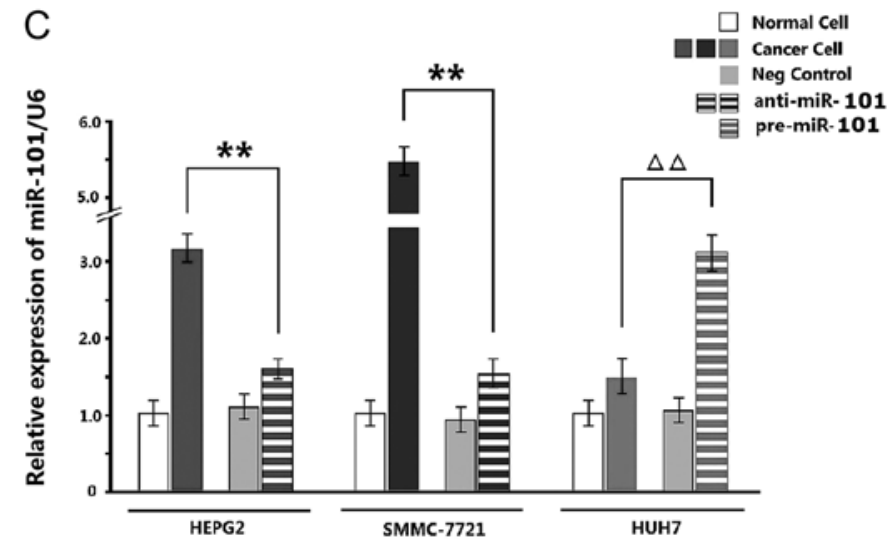

Figure 2. Expression of miR-101 in different hepatocellular carcinoma (HCC) cell lines. (A) A total of 466 genes underwent significantly different changes according to the results of digital gene expression profiling, and target genes of miR-101 were predicted by miRBase (bottom). CXCL12, IL6R, FOXO3 and phosphatase and tensin homolog deleted on chromosome 10 (PTEN) were the desired genes (right); (B) qPCR detection showed the levels of HepG2 and SMMC-7721 cells were upregulated maximally, while that of Huh7 cells was upregulated minimally; (C) miR-101 expression levels in HepG2 and SMMC-7721 cells were significantly decreased after being transfected with miR-101 inhibitor $(\mathrm{P}<0.01)$, while that of Huh7 cells was increased after being transfected with miR-101 mimics $(\mathrm{P}<0.01) .{ }^{* *} \mathrm{P}<0.01$.

predicted as the target genes of miR-101 by miRBase. Hence, CXCL12, IL6R, FOXO3 and PTEN were determined as the desired genes based on the two tests (Fig. 2A).

qPCR detection exhibited that miR-101 expression levels in $8 \mathrm{HCC}$ cell lines were upregulated compared with that in normal cell line L-02. Especially, the levels of HepG2 and SMMC-7721 cells were upregulated maximally, while that of Huh7 cells was upregulated minimally (Fig. 2B). Therefore, miR-101 inhibitor was added in HepG2 and SMMC-7721 cells, and miR-101 mimics were added in Huh7 cells. Compared with NC and blank groups, the three kinds of cells underwent significant changes after being transiently transfected with inhibitor and mimics $(\mathrm{P}<0.01)$ (Fig. 2C).

Determination of miR-101 desired genes by qPCR and Western blotting. Of the 4 potential target genes, only PTEN gene was expressed significantly differently, as suggested by qPCR results. Adding miR-101 inhibitor significantly increased the PTEN expressions in HepG2 and SMMC-7721 cells than that in the NC control group, whereas adding miR-101 mimics led to significant decrease in Huh7 cells (Fig. 3A). In contrast, CXCL12, IL6R and FOXO3 did not experience the same changes.

Western blotting also exhibited that only PTEN protein was significantly upregulated $(\mathrm{P}<0.05)$ or downregulated $(\mathrm{P}<0.01)$ (Fig. 3B and C).

Verification of PTEN by luciferase reporter assay. Twelve sequences of miR-101, which were identical to the WT-PTEN-3'UTR end, were disrupted by the MUT-PTEN-3'UTR (Fig. 4A). The luciferase reporter assay of $293 \mathrm{~T}$ cells showed that negative control pGL3M-MUT-PTEN-3'UTR and pGL3M-WT-PTEN-3'UTR 


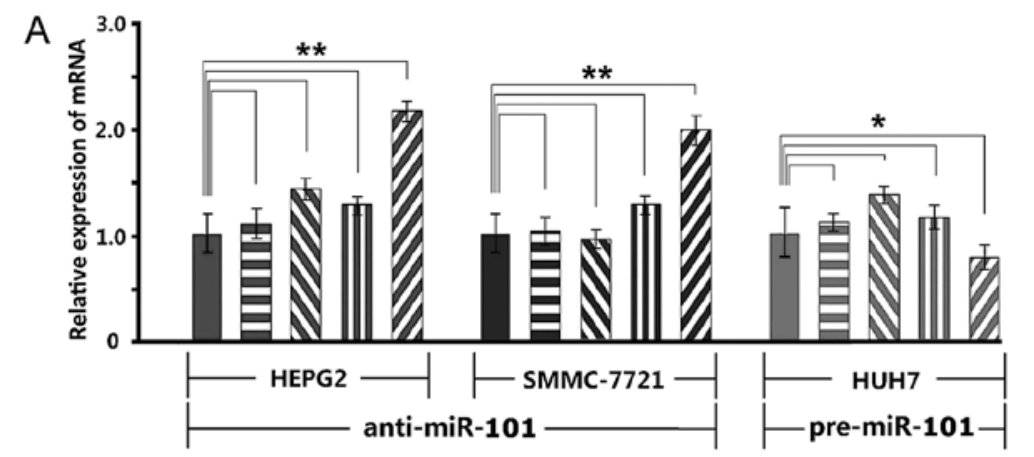

$\square \square \square$ NC

ECXCL12 $\mathbf{N}$ IL6R

IIIFOXO3 VI PTEN
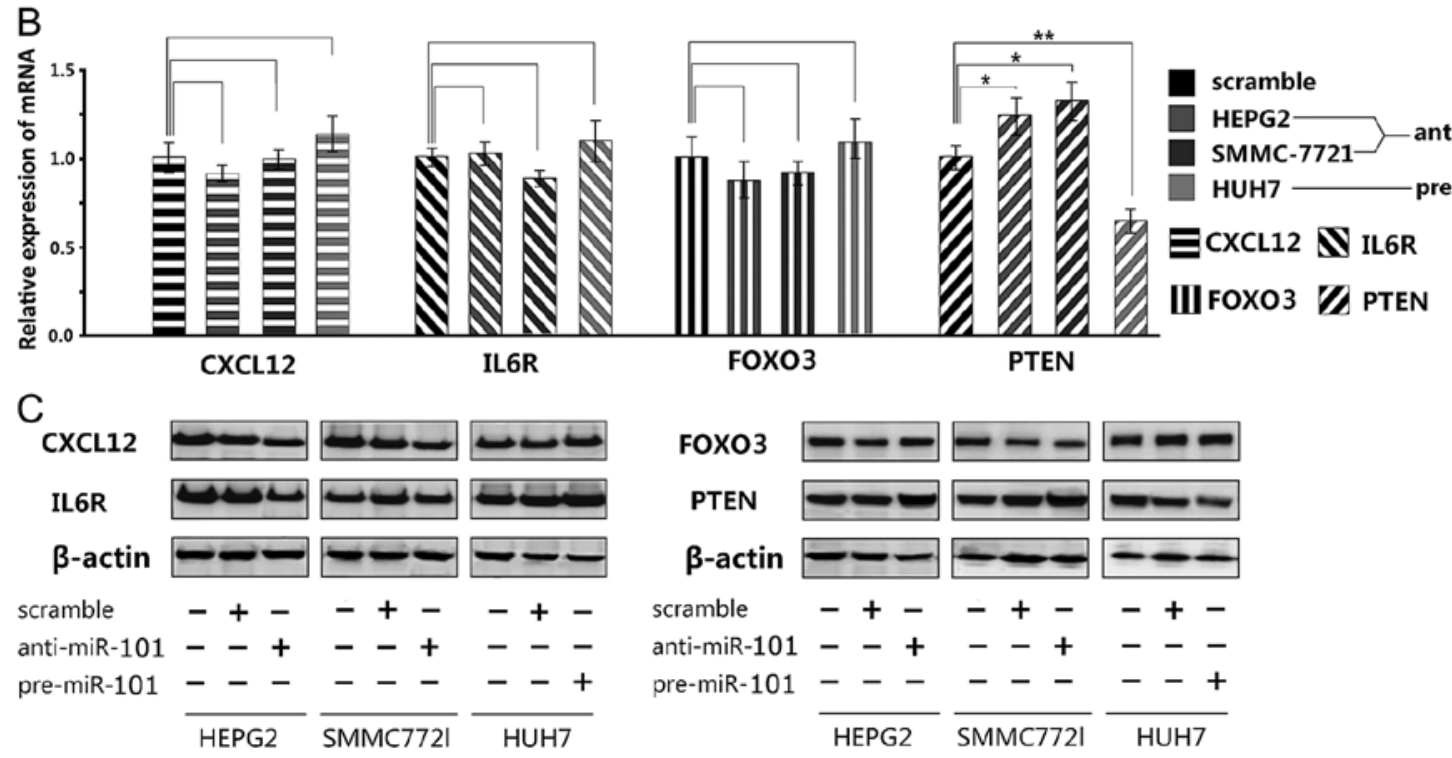

Figure 3. Determination of miR-101 desired genes by qPCR and western blotting. (A) qPCR results showed that of the 4 potential target genes, only phosphatase and tensin homolog deleted on chromosome 10 (PTEN) gene was expressed significantly differently; (B and C) western blotting exhibited that only PTEN protein was significantly upregulated $(\mathrm{P}<0.05)$ or downregulated $(\mathrm{P}<0.01) .{ }^{* *} \mathrm{P}<0.01,{ }^{*} \mathrm{P}<0.05$.

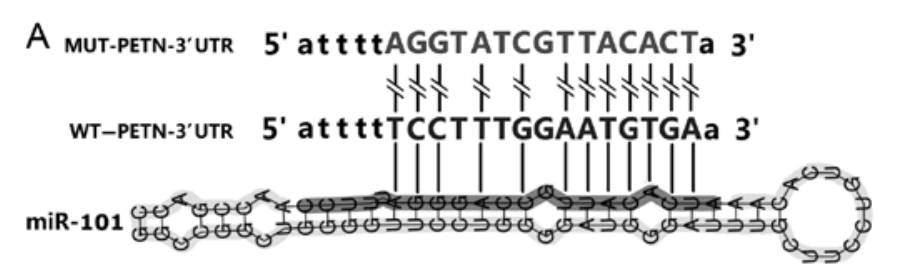

B

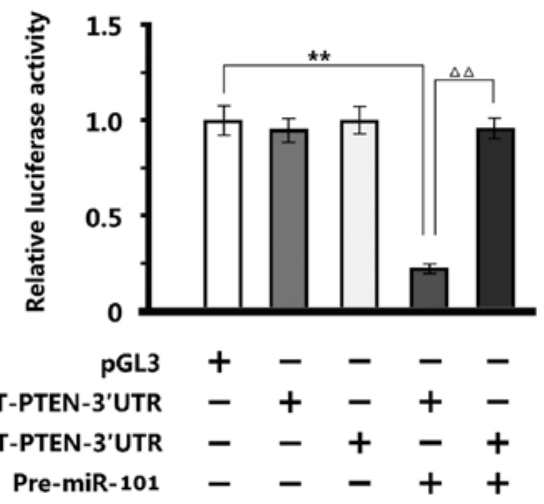

Figure 4. Verification of phosphatase and tensin homolog deleted on chromosome 10 (PTEN) by luciferase reporter assay. (A) Twelve sequences of miR-101, which were identical to WT-PTEN-3'UTR end, were disrupted by MUT-PTEN-3'UTR; (B) luciferase reporter assay of 293T cells showed that negative control pGL3M-MUT-PTEN-3'UTR and pGL3M-WT-PTEN-3'UTR were similar to empty plasmid pGL3M. Adding miR-101 mimics did not alter the activity of MUT group significantly, but significantly reduced the fluorescent intensity of WT group. ${ }^{* *} \mathrm{P}<0.01$. were similar to empty plasmid pGL3M. Adding miR-101 mimics did not alter the activity of MUT group significantly, but significantly reduced the fluorescent intensity of WT group, indicating that miR-101 bound and changed the specific sequences of WT-PTEN-3'UTR promoter (Fig. 4B).

\section{Discussion}

By using digital miRNA expression profiling, we herein observed that miR-101 was significantly upregulated. Besides, qPCR detection showed that miRNA expression in tumor tissues was upregulated by over 2 -fold. The expressions of miR-101 in SMMC-7721 and HepG2 cells were highly expressed, and that in Huh7 cells was lowly expressed. Therefore, the three types of cells were used (28).

Since miR-101 was ubiquitously highly expressed in HCC cells, the results of digital miRNA expression profiling were verified correctly. Moreover, correlation between miR-101 expression and clinical prognosis of HCC was also studied. Although miR-101 has been correlated with the prognosis of malignant tumors previously, it has rarely been correlated with that of HCC (5). Upregulation of miR-101 was the independent prognostic factor of overall and recurrence-free survival rates. High miR-101 expression was correlated with poor prognosis independent of tumor staging and other clinical variables, suggesting that miR-101 is a promising molecular marker for 
determining the prognosis of HCC. In this study, HCC metastasis-associated miRNAs were correlated with recurrence and survival rates, and the expression levels of most miRNAs were correlated with survival time, being consistent with previous studies (29-31).

Of the four candidate genes, only PTEN yielded desirable results. Ever since 1997, PTEN, mutated in multiple advanced cancers 1 (MMAC1) and TGF- $\beta$-regulated and epithelial cell-enriched phosphatase (TEP1) were found $(32,33)$ which are actually the same gene that is referred to as PTEN now. PTEN is one of the genes that are most prone to mutation, the deletion and mutation of which are bound to induce malignant glioma, prostate cancer and HCC $(34,35)$. As a highly conservative gene, PTEN affects many types of tumors by bispecific lipase.

Anti-miR-101 regulates the transcription of PTEN that influences the growth, apoptosis, proliferation and adhesion of tumor cells by mainly regulating negatively the PDK/AKT signaling pathway. Whereas, the PI3K/PKB/AKT signaling transduction pathway mainly promotes cell proliferation (36). PTEN protein can antagonize the effects of phosphatidylinositol PI3K by dephosphorylating the substrate, and decreasing the contents of second messengers phosphatidylinositol3,4-diphosphate and phosphatidylinositol $(3,4,5)$-triphosphate. Thus, the activity of protein kinase can be maintained normal after negative regulation of the PI3K/PKB/AKT signaling transduction pathway, thus regulating cell proliferation, differentiation and apoptosis. Hence, this pathway is able to suppress cancer (37), by which anti-miR-101 may exert inhibitory effects. It has previously been reported that miR-101 may regulate cell proliferation, differentiation and apoptosis by affecting mitogen-activated protein kinase (MAPK) via a certain pathway (38).

As the first anti-oncogene with phosphatase activity, PTEN inspires structural and functional studies for potential malignant tumor treatment. Mutter et al diagnosed early endometrial cancer by using PTEN as a marker (39). In 2011, RNA was associated with cancer onset by manipulating the fate of molecules $(12,13)$. In case the dialogue between PTEN gene and miRNA is ruined, unexpected disasters may occur. On the other hand, altering the dialogue may be able to prevent and treat cancer (40). The RNA management network seems to be applicable to non-protein-coding regions, thus playing an essential role in maintaining normal muscle development.

Although the correlation between anti-miR-101 and PTEN has been reported $(40,41)$, in vitro cell experiments and clinical studies have seldom been referred simultaneously. We herein verified that high miR-101 expression may facilitate the proliferation of HCC cells and miR-101 may participate in the onset of HCC as an oncogene. Moreover, the impact of miR-101 on PTEN was also demonstrated, being in accordance with the results of Karreth et al (11). In conclusion, anti-miR-101 may function as a small-molecule antitumor agent for HCC, and this study provides evidence for clarifying the molecular mechanism of HCC onset.

\section{References}

1. Fang M, Zhao YP, Zhou FG, Lu LG, Qi P, Wang H, Zhou K, Sun SH, Chen CY and Gao CF: N-glycan based models improve diagnostic efficacies in hepatitis B virus-related hepatocellular carcinoma. Int J Cancer 127: 148-159, 2010.
2. Pang RW, Joh JW, Johnson PJ, Monden M, Pawlik TM and Poon RT: Biology of hepatocellular carcinoma. Ann Surg Oncol 15: 962-971,2008.

3. Shao Q, Ren P, Li Y, Peng B, Dai L, Lei N, Yao W, Zhao G, Li L and Zhang J: Autoantibodies against glucose-regulated protein 78 as serological diagnostic biomarkers in hepatocellular carcinoma. Int J Oncol 41: 1061-1067, 2012.

4. Wang L, Li B, Li L and Wang T: MicroRNA-497 suppresses proliferation and induces apoptosis in prostate cancer cells. Asian Pac J Cancer Prev 14: 3499-3502, 2013.

5. Su H, Yang JR, Xu T, Huang J, Xu L, Yuan Y and Zhuang SM: MicroRNA-101, down-regulated in hepatocellular carcinoma, promotes apoptosis and suppresses tumorigenicity. Cancer Res 69: 1135-1142, 2009.

6. Saumet A, Vetter G, Bouttier M, Antoine E, Roubert C, Orsetti B, Theillet $\mathrm{C}$ and Lecellier $\mathrm{CH}$ : Estrogen and retinoic acid antagonistically regulate several microRNA genes to control aerobic glycolysis in breast cancer cells. Mol Biosyst 8: 3242-3253, 2012.

7. Varambally S, Cao Q, Mani RS, Shankar S, Wang X, Ateeq B, Laxman B, Cao X, Jing X, Ramnarayanan K, et al: Genomic loss of microRNA-101 leads to overexpression of histone methyltransferase EZH2 in cancer. Science 322: 1695-1699, 2008

8. Saito A, Suzuki HI, Horie M, Ohshima M, Morishita Y, Abiko Y and Nagase T: An integrated expression profiling reveals target genes of TGF- $\beta$ and TNF- $\alpha$ possibly mediated by microRNAs in lung cancer cells. PLoS One 8: e56587, 2013.

9. Piepoli A, Tavano F, Copetti M, Mazza T, Palumbo O, Panza A, di Mola FF, Pazienza V, Mazzoccoli G, Biscaglia G, et al: Mirna expression profiles identify drivers in colorectal and pancreatic cancers. PLoS One 7: e33663, 2012.

10. Fulci V, Chiaretti S, Goldoni M, Azzalin G, Carucci N, Tavolaro S, Castellano L, Magrelli A, Citarella F, Messina M, et al: Quantitative technologies establish a novel microRNA profile of chronic lymphocytic leukemia. Blood 109: 4944-4951, 2007.

11. Karreth FA, Tay Y, Perna D, Ala U, Tan SM, Rust AG, DeNicola G, Webster KA, Weiss D, Perez-Mancera PA, et al: In vivo identification of tumor- suppressive PTEN ceRNAs in an oncogenic BRAF-induced mouse model of melanoma. Cell 147: 382-395, 2011.

12. Tay Y, Kats L, Salmena L, Weiss D, Tan SM, Ala U, Karreth F, Poliseno L, Provero P, Di Cunto F, et al: Coding-independent regulation of the tumor suppressor PTEN by competing endogenous mRNAs. Cell 147: 344-357, 2011

13. Lee SS, Shin HS, Kim HJ, Lee SJ, Lee HS, Hyun KH, Kim YH, Kwon BW, Han JH, Choi $\mathrm{H}$, et al: Analysis of prognostic factors and 5-year survival rate in patients with hepatocellular carcinoma: A single-center experience. Korean J Hepatol 18: 48-55, 2012.

14. Ang SF, Tan SH, Toh HC, Poon DY, Ong SY, Foo KF and Choo SP: Activity of thalidomide and capecitabine in patients with advanced hepatocellular carcinoma. Am J Clin Oncol 35: 222-227, 2012.

15. Zhou L, Rui JA, Wang SB, Chen SG and Qu Q: Risk factors of poor prognosis and portal vein tumor thrombosis after curative resection of solitary hepatocellular carcinoma. Hepatobiliary Pancreat Dis Int 12: 68-73, 2013.

16. Zhang Y, Deng ZS, Liao MM, Wang N, Zhang XQ, Yu HY and Zhang YD: Tumor associated glycoprotein-72 is a novel marker for poor survival in hepatocellular carcinoma. Pathol Oncol Res 18: 911-916, 2012.

17. Yang W, Sun T, Cao J, Liu F, Tian Y and Zhu W: Downregulation of miR-210 expression inhibits proliferation, induces apoptosis and enhances radiosensitivity in hypoxic human hepatoma cells in vitro. Exp Cell Res 318: 944-954, 2012.

18. Guo J, Yao F, Lou Y, Xu C, Xiao B, Zhou W, Chen J, Hu Y and Liu Z: Detecting carcinoma cells in peripheral blood of patients with hepatocellular carcinoma by immunomagnetic beads and rt-PCR. J Clin Gastroenterol 41: 783-788, 2007.

19. Zheng X, Pan C, Diao Y, You Y, Yang C and Hu Z: Development of microsatellite markers by transcriptome sequencing in two species of Amorphophallus (Araceae). BMC Genomics 14: 490, 2013.

20. Xia H, Zhao C, Hou L, Li A, Zhao S, Bi Y, An J, Zhao Y, Wan S and Wang X: Transcriptome profiling of peanut gynophores revealed global reprogramming of gene expression during early pod development in darkness. BMC Genomics 14: 517, 2013.

21. Gorur A, Balci Fidanci S, Dogruer Unal N, Ayaz L, Akbayir S, Yildirim Yaroglu H, Dirlik M, Serin MS and Tamer L: Determination of plasma microRNA for early detection of gastric cancer. Mol Biol Rep 40: 2091-2096, 2013. 
22. Burgucu D, Guney K, Sahinturk D, Ozbudak IH, Ozel D, Ozbilim $G$ and Yavuzer $U$ : Tbx3 represses PTEN and is over-expressed in head and neck squamous cell carcinoma. BMC Cancer 12: 481, 2012.

23. Ekizoglu S, Dalay N, Karaman E, Akdeniz D, Ozaydin A and Buyru N: LKB1 downregulation may be independent of promoter methylation or FOXO3 expression in head and neck cancer. Transl Res 162: 122-129, 2013.

24. de Oliveira KB, Guembarovski RL, Guembarovski AM, da Silva do Amaral Herrera AC, Sobrinho WJ, Ariza CB and Watanabe MA: CXCL12, CXCR4 and IFN $\gamma$ genes expression: Implications for proinflammatory microenvironment of breast cancer. Clin Exp Med 13: 211-219, 2013.

25. Tsukamoto K, Ohta N, Shirai Y and Emi M: A highly polymorphic CA repeat marker at the human interleukin 6 receptor (IL6R) locus. J Hum Genet 43: 289-290, 1998

26. Fulzele A, Malgundkar SA, Govekar RB, Patil A, Kane SV, Chaturvedi P, D'Cruz AK and Zingde SM: Proteomic profile of keratins in cancer of the gingivo buccal complex: Consolidating insights for clinical applications. J Proteomics 91: 242-258, 2013.

27. Dou LP, Li YH, Wang LL and Yu L: HOXA9 is direct target of miR-196a. Xi Bao Yu Fen Zi Mian Yi Xue Za Zhi 27: 166-169, 2011 (In Chinese).

28. Wang YP, Tang XJ, Chen XH, Che GW, Zhu DX, Sun ZL and Zhou QH: Study on cloning of hTERT promoter and its targeting transcriptional activities in telomerase-positive lung cancer cells. Sichuan Da Xue Xue Bao Yi Xue Ban 37: 497-501, 2006 (In Chinese).

29. Zhi Q, Zhu J, Guo X, He S, Xue X, Zhou J, Hu B, Li H, Chen S, Zhao H, et al: Metastasis-related miR-185 is a potential prognostic biomarker for hepatocellular carcinoma in early stage. Biomed Pharmacother 67: 393-398, 2013.

30. Katayama Y, Maeda M, Miyaguchi K, Nemoto S, Yasen M, Tanaka S, Mizushima H, Fukuoka Y, Arii S and Tanaka H: Identification of pathogenesis-related microRNAs in hepatocellular carcinoma by expression profiling. Oncol Lett 4: 817-823, 2012.

31. Kim WH, Min KT, Jeon YJ, Kwon CI, Ko KH, Park PW Hong SP, Rim KS, Kwon SW, Hwang SG, et al: Association study of microRNA polymorphisms with hepatocellular carcinoma in Korean population. Gene 504: 92-97, 2012.
32. Furnari FB, Lin H, Huang HS and Cavenee WK: Growth suppression of glioma cells by PTEN requires a functional phosphatase catalytic domain. Proc Natl Acad Sci USA 94: 12479-12484, 1997.

33. Dahia PL, Marsh DJ, Zheng Z, Zedenius J, Komminoth P, Frisk T, Wallin G, Parsons R, Longy M, Larsson C, et al: Somatic deletions and mutations in the Cowden disease gene, PTEN, in sporadic thyroid tumors. Cancer Res 57: 4710-4713, 1997.

34. Inaba N, Kimura M, Fujioka K, Ikeda K, Somura H, Akiyoshi K, Inoue Y, Nomura M, Saito Y, Saito H, et al: The effect of PTEN on proliferation and drug-, and radiosensitivity in malignant glioma cells. Anticancer Res 31: 1653-1658, 2011.

35. Barbosa M, Henrique M, Pinto-Basto J, Claes K and Soares G: Prostate cancer in Cowden syndrome: Somatic loss and germline mutation of the PTEN gene. Cancer Genet 204: 224-225, 2011.

36. Ogita $\mathrm{S}$ and Lorusso P: Targeting phosphatidylinositol 3 kinase (PI3K)-Akt beyond rapalogs. Target Oncol 6: 103-117, 2011.

37. Karra L, Shushan A, Ben-Meir A, Rojansky N, Klein BY, Shveiky D, Levitzki R and Ben-Bassat $\mathrm{H}$ : Changes related to phosphatidylinositol 3-kinase/Akt signaling in leiomyomas: Possible involvement of glycogen synthase kinase 3alpha and cyclin D2 in the pathophysiology. Fertil Steril 93: 2646-2651, 2010.

38. Kim HA, Kim KJ, Seo KH, Lee HK and Im SY: PTEN/MAPK pathways play a key role in platelet-activating factor-induced experimental pulmonary tumor metastasis. FEBS Lett 586: 4296-4302, 2012.

39. Mutter GL, Lin MC, Fitzgerald JT, Kum JB, Baak JP, Lees JA, Weng LP and Eng C: Altered PTEN expression as a diagnostic marker for the earliest endometrial precancers. J Natl Cancer Inst 92: 924-930, 2000.

40. Chen Y, Sun Y, Chen L, Xu X, Zhang X, Wang B, Min Land Liu W: miRNA-200c increases the sensitivity of breast cancer cells to doxorubicin through the suppression of E-cadherin-mediated PTEN/Akt signaling. Mol Med Rep 7: 1579-1584, 2013.

41. Xiong X, Ren HZ, Li MH, Mei JH, Wen JF and Zheng CL: Down-regulated miRNA-214 induces a cell cycle G1 arrest in gastric cancer cells by up-regulating the PTEN protein. Pathol Oncol Res 17: 931-937, 2011. 